\title{
Single-Phase Source-Coupled Adiabatic Logic
}

\author{
Suhwan Kim and Marios C. Papaefthymiou \\ Advanced Computer Architecture Laboratory \\ Department of Electrical Engineering and Computer Science \\ University of Michigan \\ Ann Arbor, MI 48109
}

\begin{abstract}
Adiabatic circuits offer a promising alternative to conventional circuitry for low energy design. Their operation is nevertheless subject to fundamental energy-speed trade-offs, just like any other physical realization of boolean logic. Thus, adiabatic circuits with very low energy consumption at low frequencies fail to function at high operating frequencies. Conversely, high-speed adiabatic circuits tend to be dissipative at low clock rates.

This paper describes SCAL, a single-phase source-coupled adiabatic logic family that operates efficiently across a wide range of operating frequencies. In layout-based simulations with $0.5 \mu \mathrm{m}$ CMOS process parameters, pipelined carry-lookahead adders developed in our logic function correctly from $10 \mathrm{MHz}$ up to $280 \mathrm{MHz}$. Our SCAL adders are less dissipative than corresponding designs in alternative adiabatic families that remain functional across the same frequency range. Moreover, they are about as dissipative as other adiabatic circuits that are geared towards very efficient operation at low frequencies. In comparison with their CMOS counterparts, our SCAL adders are 3 to 10 times more energy efficient.
\end{abstract}

\section{Introduction}

Adiabatic circuit architectures reduce energy dissipation by steering currents across devices with low voltage differences and by recycling the energy stored in their capacitors [2,3]. Broadly speaking, the efficient operation of these designs is yet another manifestation of energy-speed trade offs. Consequently, adiabatic circuits that operate very efficiently at low operating frequencies stop functioning at high data rates. On the other hand, adiabatic circuits with broad operating ranges tend to be dissipative at low frequencies.

This paper presents a source-coupled adiabatic logic family, called SCAL, that achieves very low dissipation across a wide range of operating frequencies. High speed is ensured by activating a sense-amplifier structure in a nonadiabatic manner. High energy efficiency across a broad frequency range is achieved by providing each gate with a current source that can be tuned by transistor sizing to achieve optimal charging rates for its operating conditions. In addition to low energy consumption and broad operating spectrum, SCAL features include true single-phase operation, balanced clock loading, functional completeness, and straightforward cascading.

A plethora of adiabatic logic families has been proposed in recent years. Several of these families, including 2N-2P, 2N-2N2P, PAL, CAL, and TSEL, use a sense-amplifier structure to drive their outputs adiabatically $[4,6,7,8,9]$. The logic family we present in this paper is an enhancement of TSEL, a true single-phase adiabatic logic. SCAL achieves increased energy efficiency across a broad frequency range while at the same time maintaining all the positive aspects of TSEL.

The remainder of this paper has four sections. The structure and operation of our logic is described in Section 2. Section 3 describes the operation of cascaded SCAL gates. Section 4 presents simulation results from layouts of pipelined 4-bit CLAs that were designed using a $0.5 \mu \mathrm{m}$ standard CMOS technology. Our contributions and ongoing research are summarized in Section 5 .

\section{SCAL Structure and Operation}
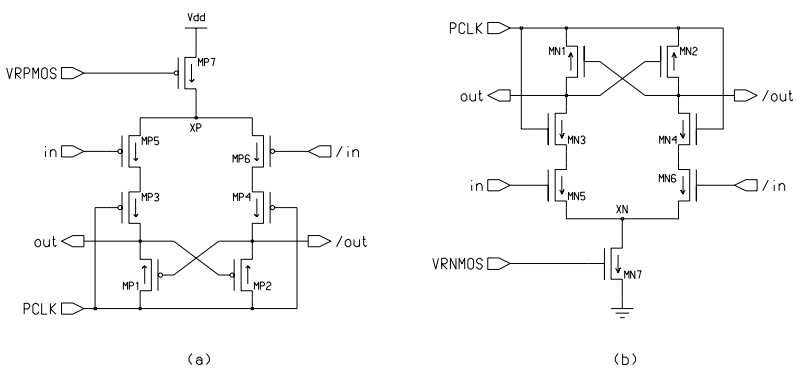

Figure 1: (a) A PMOS and (b) an NMOS inverter in SCAL.

SCAL is a partially-adiabatic logic family that is clocked by a single-phase power-clock. Low energy operation is achieved across a broad operating range by tuning a current source attached to each gate. The basic structure of a SCAL PMOS gate is shown in the PMOS inverter of Figure 1(a). This inverter comprises a pair of cross-coupled latches (MP1 and MP2), a pair of current control switches (MP3 and MP4), two function blocks (MP5 and MP6) and a current source (MP7). This current source is the main structural characteristic that differentiates SCAL from TSEL, its closest adiabatic relative. The charge flow rate through the current source is controlled by the $W / L$ ratio of MP7. The constant voltage supply $V_{d d}$ is required for activating the cross-coupled latches. The port PCLK is used to apply a sinusoidal power-clock $\Phi$.

A PMOS SCAL gate operates in two phases: discharge and evaluate. The energy stored in the node out or $\overline{\text { out }}$ is recovered during discharge. In this phase, the power-clock $\Phi$ starts from high and ramps down toward low, pulling both out and out down toward the PMOS threshold voltage $\left|V_{t p}\right|$. The new output of a PMOS gate is computed during evaluate. In this phase, $\Phi$ starts rising from low, turning MP1 and MP2 on along the way. When the gateto-source voltage $\left|V_{g s p}\right|$ of the current source MP7 exceeds $\left|V_{t p}\right|$, MP7 turns on and raises the voltage $V_{\text {xp }}$ of the internal node XP. While $\Phi$ remains below $V_{\mathrm{xp}}-\left|V_{t p}\right|, \mathrm{MP} 3$ and MP4 are conducting. Therefore, assuming that in is high and in is low, a pull-up path is created from $V_{\text {xp }}$ to $\overline{\text { out }}$, and the voltage at out starts rising toward $V_{\mathrm{xp}}$. The cross-coupled latches function as a sense-amplifier and boost the voltage difference of the two output nodes. As soon as this difference exceeds $\left|V_{t p}\right|$, MP1 turns off and the output out is charged adiabatically from that point on. When $\Phi$ exceeds $V_{\mathrm{xp}}-$ $\left|V_{t p}\right|, \mathrm{MP} 3$ and MP4 turn off and disconnect the functional blocks 
from the outputs out and $\overline{\text { out }}$. Hence, any further changes in the inputs do not propagate to the outputs. By the end of the evaluation phase, $\overline{\text { out }}$ is charged up to the peak power-clock voltage.

The impact of the various circuit parameters on SCAL operation can be best understood by examining the equation describing the current of a MOS device in its saturation region:

$$
I_{d s}=\frac{1}{2} \mu C_{o x}(W / L)\left(V_{g s}-V_{t}\right)^{2}, \quad V_{d s}>V_{g s}-V_{t}>0
$$

where $I_{d s}$ is the drain-to-source current, $V_{g s}$ is the gate-to-source voltage, $V_{t}$ is the device threshold voltage, $\mu$ is the effective surface mobility of the carrier in the channel, and $C_{o x}$ is the gate-oxide capacitance. From this equation, it follows that the current through the current source is controlled by the $W / L$ ratio and $V_{g s}$ [1]. For low-energy operation, the duration of evaluate should be made as short as possible by appropriately selecting the biasing voltage of the current sources. For high-speed operation, the source current should be made large enough by appropriate transistor sizing.

The main difference in the operation of SCAL and TSEL is in the control of the evaluation phase. In TSEL, a reference voltage is used to control both the duration of the evaluation phase and the value of the charging current. To ensure correct function, however, the reference voltages must be two times larger than the thresholds of the PMOS and NMOS devices. Thus, at low frequencies, the duration of evaluate cannot be shortened below a certain limit, and TSEL dissipation increases even when multiple reference voltages are used. On the other hand, the current flow in SCAL can be controlled by adjusting the $W / L$ ratio of MP7, whereas the duration of evaluate is determined independently by selecting the biasing voltage of the current source. Thus, SCAL gates can be individually tuned to achieve minimum dissipation for low and high operating frequencies.

The inverter in Figure 1(b) shows the basic structure of a SCAL NMOS gate in which outputs are precharged high. The two phases in the operation of an NMOS gate are charge and evaluate.

\section{Cascading SCAL Gates}

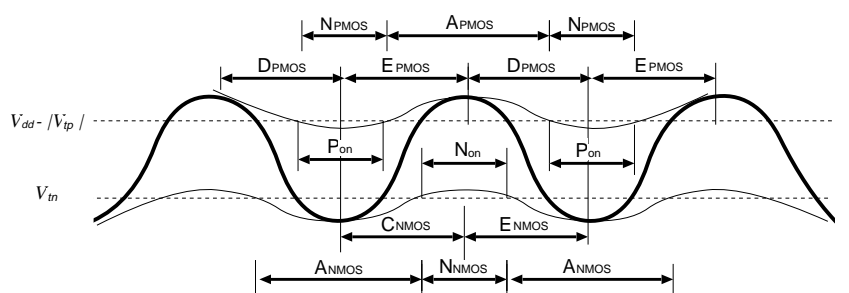

Figure 2: SCAL timing. DPMOS: PMOS discharge phase; EPMOs: PMOS evaluate phase; $P_{\text {on }}$ : PMOS current source on; $C_{\text {NMOS }}$ : NMOS charge phase; $\mathrm{E}_{\mathrm{NMOS}}$ : NMOS evaluate phase, $\mathrm{N}_{\text {on }}$ : NMOS current source on; $A_{\mathrm{PMOS}}, \mathrm{A}_{\mathrm{NMOS}}$ : adiabatic switching for PMOS and NMOS; NPMOS, N NMOS: non-adiabatic switching for PMOS and NMOS.

SCAL cascades are built by stringing together alternating PMOS and NMOS gates. The only signal required for controlling a SCAL cascade is the power-clock $\Phi$. The relative timing of the gates in a SCAL cascade is shown in Figure 2. At any time during the circuit's operation, either all PMOS gates evaluate and all NMOS gates charge or all PMOS gates discharge and all NMOS gates evaluate. While the current switches of the odd stages are off, the function blocks of the even stages are connected to $V_{d d}$ or $V_{s s}$ through the current sources and can safely evaluate their outputs. After half a cycle, while the current switches of the even stages are off, the

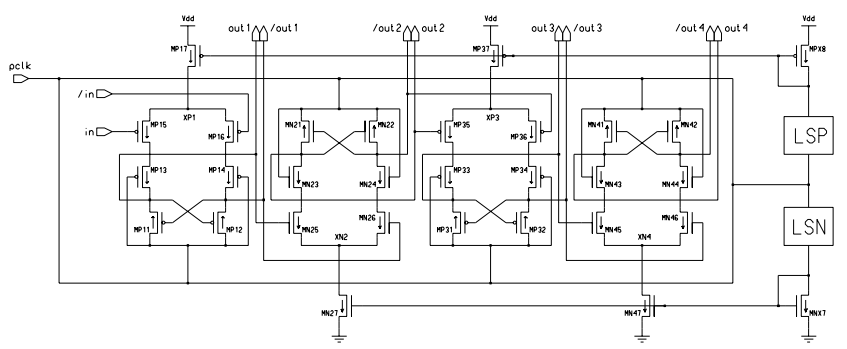

Figure 3: A pipeline of SCAL inverters with biasing circuitry.

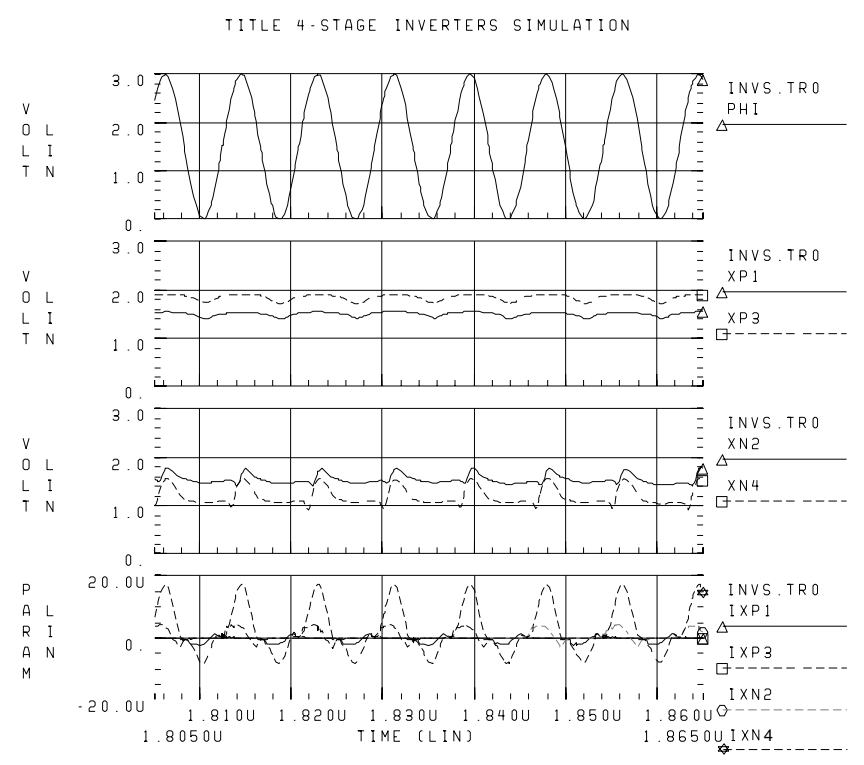

Figure 4: Waveforms obtained from HSPICE simulations of a 4stage pipeline of SCAL inverters. From top to bottom: (1) powerclock $\Phi,(2)$ internal voltages of $V_{\mathrm{xp} 1}$ and $V_{\mathrm{xp} 3}$, (3) internal voltages of $V_{\mathrm{xn} 2}$ and $V_{\mathrm{xn} 4}$, (4) currents through the current sources (devices MP17, MN27, MP37 and MN47).

function blocks of the odd stages are connected to $V_{d d}$ or $V_{s s}$ and their inputs are stable.

A PNPN cascade of SCAL inverters and the biasing circuitry for the current sources are shown in Figure 3. The blocks denoted by LSP and LSN are voltage level shifters. Figure 4 shows the internal voltages and currents through the current source for each stage of the cascade in Figure 3 when simulated at $120 \mathrm{MHz}$ with a $3 \mathrm{~V}$ peak-to-peak supply voltage and a $0.5 \mu \mathrm{m}$ standard CMOS technology. In these simulations, the $W / L$ ratio of the cross-coupled latch in each gate was $5 / 2$. For the function blocks, minimum size transistors were used with $W / L$ ratio equal to $3 / 2$. The $W / L$ ratios for the current sources (devices MP17, MN27, MP37 and MN47) were $1 / 2,1 / 4,2 / 1$ and $1 / 1$, respectively. The length of the evaluate phase is the same for every stage. The internal voltages $V_{\mathrm{xp} 1}, V_{\mathrm{xn} 2}, V_{\mathrm{xp} 3}$, and $V_{\mathrm{xn} 4}$ and the internal currents $I_{\mathrm{xp} 1}, I_{\mathrm{xn} 2}$, $I_{\mathrm{xp} 3}$, and $I_{\mathrm{xn} 4}$ are proportional to the $W / L$ ratios of the current sources. These voltages and currents affect the energy dissipation of the SCAL structures. As the $W / L$ ratio decreases, the current is reduced. Symmetrically, as the W/L ratio increases, the current associated with the non-adiabatic event in the gates increases. For each gate, the optimal ratio can be achieved independently of the duration of the non-adiabatic evaluation. Therefore, energy dissipation can be reduced by using several current source sizes as opposed to just one. 


\section{Simulation Results}

In this section, we present HSPICE simulation results for pipelined 4-bit CLAs that we developed in SCAL, TSEL, 2N-2P, PAL, and static CMOS. Our circuits were designed using a standard $0.5 \mu \mathrm{m}$ CMOS technology and were simulated with distributed RC parameters that were extracted from layout. Our simulations accounted for the dissipation of the gates and internal clock lines but did not include the energy consumed on the external clock distribution network or the power-clock generator.

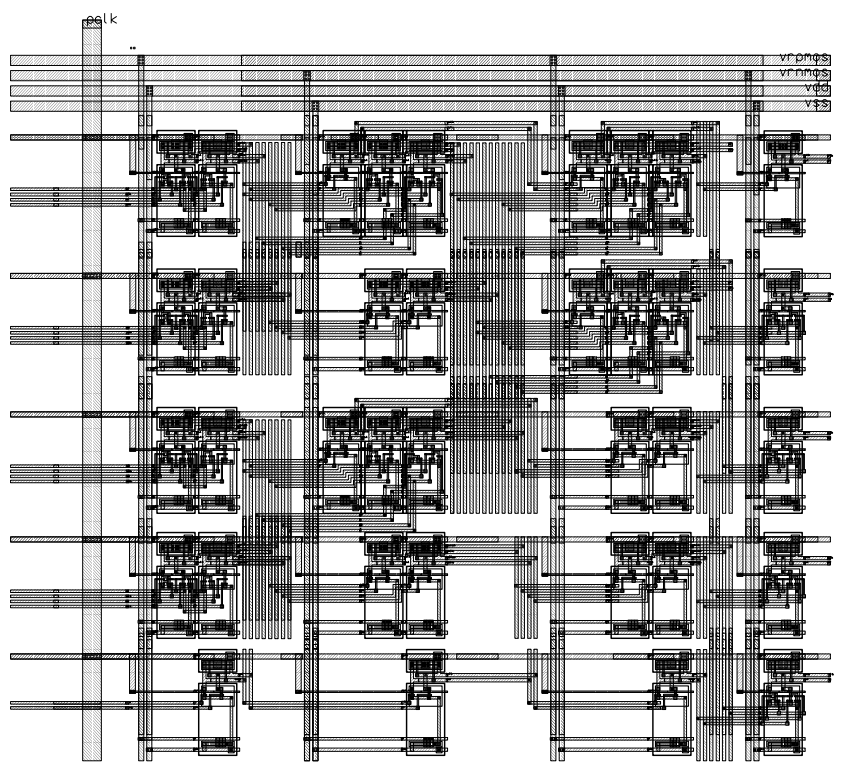

Figure 5: Layout of 4-stage pipelined 4-bit CLA in SCAL.

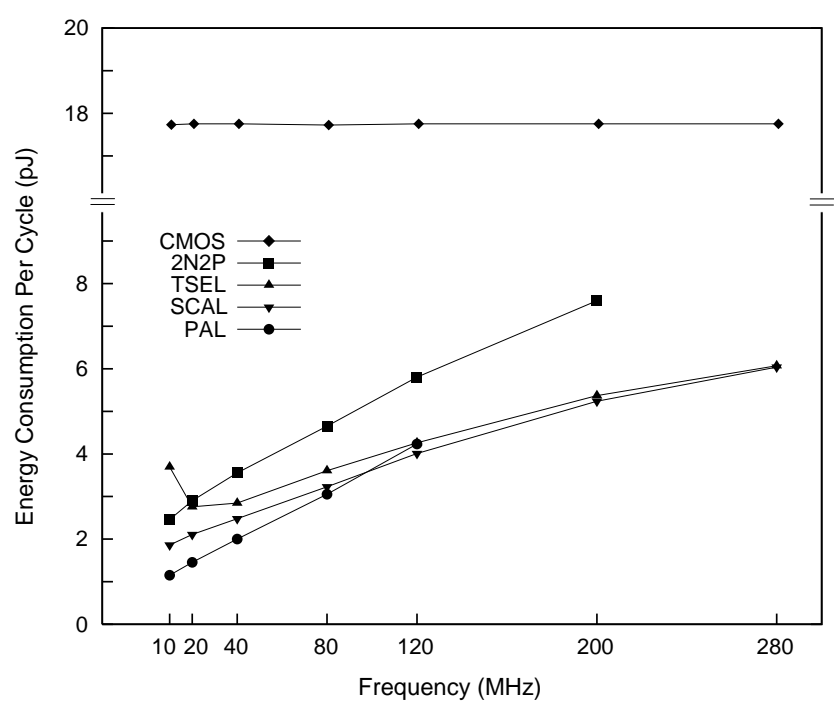

Figure 6: Energy consumption vs. frequency for 4-bit CLA.

The layout of our SCAL CLA is shown in Figure 5. The $W / L$ ratio of each current source was selected among the values $1 / 8$, $1 / 4,1 / 2$ and $1 / 1$ according to the output capacitive loads of its gate and the operating frequency. Figure 6 gives the per-cycle energy consumption of our designs as a function of their operating frequency for a uniformly distributed random input sequence. The peak power-clock voltage was $3 \mathrm{~V}$, and each primary output was connected to a $60 \mathrm{fF}$ load. As expected, the energy consumption of the static CMOS implementation does not vary with the operating frequency. SCAL is less dissipative than $2 \mathrm{~N}-2 \mathrm{P}$ and TSEL for the entire operating regime. Moreover, it is more efficient than PAL for operating frequencies above $100 \mathrm{MHz}$. Below $100 \mathrm{MHz}, \mathrm{SCAL}$ is still competitive with PAL and is no more than 50\% more dissipative at $10 \mathrm{MHz}$. SCAL operates at full logic swing for frequencies up to $280 \mathrm{MHz}$. At $280 \mathrm{MHz}$, it is about 3 times more energyefficient than static CMOS. 2N-2P breaks down at 200MHz. At that point, TSEL and SCAL are 1.5 times more efficient than $2 \mathrm{~N}-2 \mathrm{P}$.

\section{Summary and Ongoing Work}

We have described an adiabatic logic that functions efficiently across a wide operating range. Our logic requires a single power-clock phase for its operation and avoids numerous problems associated with multiple phases, including increased energy dissipation and layout complexity in the clock distribution network, clock skew, and multiple $\mathrm{AC}$ power supplies. Its efficient operation is based on a current source that can be tuned to control the rate of charge flow into or out of the gate during evaluation.

We are currently evaluating the performance of SCAL under voltage scaling [5]. Preliminary results from layout simulations of CLAs indicate that SCAL can operate with very low supply voltages and is significantly more efficient than corresponding CMOS, $2 \mathrm{~N}-2 \mathrm{P}$, TSEL, and PAL designs.

\section{Acknowledgments}

This research was supported in part by a Young Investigator Award from the US Army Research Office under Grant No. DAAG55-970395 .

\section{References}

[1] P. E. Allen and D. R. Holberg. CMOS Analog Circuit Design. Oxford University Press, 1987.

[2] W. C. Athas, L. J. Svensson, J. G. Koller, N. Tzartzanis, and Y. Chou. Low-power digital systems based on adiabatic-switching principles. IEEE Transactions on VLSI Systems, 2(4):398-406, December 1994.

[3] J. S. Denker. A review of adiabatic computing. In Proceedings of the 1994 Symposium on Low Power Electronics/Digest of Technical Papers, pages 94-97, October 1994.

[4] S. Kim and M. C. Papaefthymiou. True single-phase energy-recovering logic for low-power, high-speed VLSI. In Proceedings of International Symposium on Low-Power Electronics and Design, August 1998.

[5] S. Kim and M. C. Papaefthymiou. Low-energy adder design with a single-phase source-coupled adiabatic logic. 1999. Submitted for Publication.

[6] M. C. Knapp, P. J. Kindlmann, and M. C. Papaefthymiou. Implementing and evaluating adiabatic arithmetic units. In IEEE 1996 Custom Integrated Circuit Conference, pages 115-118, 1996.

[7] A. Kramer, J. S. Denker, B. Flower, and J. Moroney. 2nd order adiabatic computation with $2 \mathrm{~N}-2 \mathrm{P}$ and $2 \mathrm{~N}-2 \mathrm{~N} 2 \mathrm{P}$ logic circuits. In 1995 International Symposium on Low Power Design, pages 191-196, 1995.

[8] D. Maksimovic, V. G. Oklobdzija, B. Nikolic, and K. W. Current. Clocked CMOS adiabatic logic with integrated single-phase powerclock supply: Experimental results. In Proceedings of International Symposium on Low Power Electronics and Design, pages 323-327, August 1997.

[9] V. G. Oklobdzija and D. Maksimovic. Pass-transistor adiabatic logic using single power-clock supply. IEEE Transactions on Circuits and Systems-II: Analog and Digital Signal Processing, 44(10):842-846, October 1997. 